\title{
Uma experiência de aplicação de uma abordagem baseada em problemas no ensino de Teoria da Computação em sala de aula tradicional
}

\author{
Luiz Otávio Ramos Gavaza ${ }^{1}$, Laís do Nascimento Salvador ${ }^{1}$, \\ David Moises Barreto dos Santos ${ }^{2}$
}

\author{
${ }^{1}$ Departamento de Ciência da Computação - Universidade Federal da Bahia (UFBA) \\ Av. Adhemar de Barros, S/N, Ondina - 40.170-110 - Salvador - BA - Brasil \\ ${ }^{2}$ Departamento de Ciências Exatas - Universidade Estadual de Feira de Santana (UEFS) \\ Av. Transnordestina, Novo Horizonte - 44.036-900 - Feira de Santana - BA - Brasil \\ \{lgavaza,laisns\}@ufba.br, davidmbs@uefs.br
}

\begin{abstract}
There are several challenges for educators to conduct disciplines in a satisfactory manner with regard to students motivation and absorption of knowledge. In Computer Science courses, Computer Theory disciplines are an additional challenge, because they deal with topics with a high level of abstraction. This paper presents a report on the experience of applying the Problem Based Learning (PBL) approach for teaching Computer Theory disciplines. The experience was conducted in a traditional classroom using a basic infrastructure. The results of this experiment show that the students have the performance of good perceptions about an applied approach, which confirms the potential for the application of PBL in the teaching of Computer Theory.
\end{abstract}

Resumo. Diversos são os desafios dos educadores para conduzir disciplinas de forma satisfatória no que diz respeito à motivação e absorção de conhecimento pelos estudantes. Nos cursos de Computação, as disciplinas de Teoria da Computação constituem um desafio adicional, por tratarem de tópicos com alto nível de abstração. Este trabalho relata a experiência de aplicação de uma abordagem de aprendizado baseado em problemas (PBL) para o ensino da disciplina de Teoria da Computação utilizando uma infraestrutura básica. Os resultados dessa experiência mostram que os estudantes participantes tiveram boas percepções sobre a abordagem aplicada, o que confirma o potencial para aplicação de PBL no ensino de Teoria da Computação.

\section{Introdução}

Os educadores em Ciência da Computação enfrentam muitas questões no que diz respeito a como conduzir uma disciplina de forma que os estudantes estejam motivados e possam obter satisfatoriamente conhecimentos desejáveis para a sua formação. Isto não é um caso particular do ensino de Computação, mas é necessário considerar que se trata de um conhecimento relativamente novo, e que só muito recentemente está presente em instituições de ensino universitário. É necessário realizar tal consideração para argumentar os motivos pelos quais ainda não existem metodologias e procedimentos suficientemente testados. Além disto, há a natureza intrinsecamente multidisciplinar e o desenvolvimento contínuo 
da área de Computação. Embora seja possível perceber um contínuo desenvolvimento também em outras áreas do conhecimento, em Computação é notória a robustez e continuidade do progresso produzido nas últimas décadas.

O ensino de Computação em geral segue a abordagem tradicional, com educadores atuando como transmissores de conhecimento, enquanto estudantes são receptores. Ao verificar as grades curriculares dos cursos de Computação, serão encontradas algumas atividades práticas em laboratórios, sobretudo para o ensino de disciplinas com tópicos de programação e algumas atividades práticas como complemento à carga horária teórica, constituída essencialmente na exposição de conhecimento pelo educador aos estudantes. A forma de ensinar Computação pode ser determinante para o sucesso também no que diz respeito a inclusão do estudantes, esse é um motivo a mais de estímulo para que os educadores pesquisem sobre essa questão.

Na Universidade Federal da Bahia (UFBA), na grade do curso de Sistema de Informação, tem-se a disciplina introdutória de Teoria da Computação, que possui uma carga teórica extensa, que além dos tópicos de Linguagens Formais e Teoria da Computação, também incluí tópicos de Complexidade e de Compiladores. Nessa disciplina, os tópicos tratados possuem um alto nível de abstração e exigem bastante esforço por parte dos estudantes para que possam acompanhar o seu andamento. As disciplinas de Teoria da Computação normalmente possuem um alto índice de reprovação e evasão. Muitas são as dificuldades para que os estudantes se sintam estimulados e consigam uma absorção satisfatória dos conceitos.

Esse trabalho relata a experiência de ensino dessa disciplina de Computação com uma abordagem de aprendizado baseado em problemas, que será referenciada nesse texto pela sigla PBL ${ }^{1}$. A metodologia PBL pode ser definida como uma abordagem educacional construtivista em que o foco de aprendizagem está nos estudantes, que são capacitados para que assumam a responsabilidade pela aprendizagem [Dolmans et al. 2005]. Na metodologia PBL o problema é uma ferramenta que fornece aos estudantes motivações para que eles alcancem os objetivos de aprendizagem [Wood 2003, O'Grady 2012]. A metodologia PBL não se resume à resolução de problema em si, mas usá-lo como ferramenta para estimular o estudante e favorecer a compreensão dos conceitos [Wood 2003].

Além desta seção introdutória, este trabalho está organizado da seguinte forma: a Seção 2 para descrever a experiência; a Seção 3 para apresentar os trabalhos correlatos; a Seção 4 para trazer uma discussão sobre alguns dos resultados obtidos com a experiência; por fim, a Seção 5 para trazer algumas das argumentações sobre consequências dos resultados exibidos na Seção anterior, as expectativas e as possibilidades de trabalhos na investigação proposta.

\section{Descrição da experiência}

Aprendizagem baseada em problemas é utilizada em muitas escolas de medicina, onde já existe alguma consolidação da abordagem como efetiva para educação médica, enquanto em outras áreas do conhecimento há tentativas de explorar as suas potencialidades. No ensino de Computação, a aplicação de PBL ainda é restrita, e geralmente acontece por iniciativa própria de alguns educadores, quase sempre isoladamente tentando melhorar a absorção do conteúdo de uma disciplina pelos estudantes [Wood 2003, O'Grady 2012].

\footnotetext{
${ }^{1}$ Acrônimo para a denominação mais difundida: Problem Based Learning.
} 
A utilização da metodologia PBL não apenas permite desenvolvimento técnico dos participantes, mas também os capacita em outros atributos, como comunicação, trabalho em equipe, resolução de problemas, autodidatismo, compartilhamento de conhecimento e informações, argumentação e respeito às divergências [Wood 2003].

A experiência de aplicação desta abordagem se deu em uma disciplina na Universidade Federal da Bahia que apresenta os conceitos introdutórios de Teoria da Computação para estudantes de graduação. A carga horária de 68 horas da disciplina no semestre foi dividida em: 26 horas de sessões tutoriais de PBL; 32 horas de aulas para apresentação de conteúdo pelo educador utilizando a abordagem tradicional; e 10 horas para aplicação de avaliações tradicionais.

Uma sessão tutorial é um período de tempo disponibilizado para que os estudantes discutam presencialmente sobre um problema e cada sessão tutorial tem duração de 2 horas. Uma sessão tutorial pode ser conduzida de várias formas, sendo que uma sessão típica é composta por um pequeno grupo de estudantes e um tutor para facilitar o andamento da sessão.

Os participantes dessa experiência foram todos os vinte e cinco estudantes regularmente inscritos na disciplina. A experiência aconteceu em uma sala de aula tradicional equipada com um quadro branco. Os estudantes foram distribuídos em semicírculo para que todos fossem capazes de ver o que era escrito no quadro e facilitar a interação entre eles.

O primeiro passo a cada sessão é a escolha dentre os estudantes de três voluntários. Um voluntário é responsável por realizar o registro da discussão da sessão no quadro branco, esse participante é denominado relator de quadro. Um segundo voluntário é responsável por realizar o registro das discussões e disponibilizar um documento consolidado para todos os participantes logo após a sessão, sendo denominado relator de mesa. A discussão é conduzida por um terceiro voluntário, denominado coordenador da sessão, que administra as intervenções dos participantes, permitindo que esses tenham espaço para se posicionar. Há um rodízio de papéis a cada sessão para que todos os estudantes tenham oportunidade de desempenhar todos os papéis descritos acima.

O tutor, por sua vez, é o principal responsável por facilitar andamento da sessão, zelando pelos objetivos de aprendizagem. Sendo um agente passivo da sessão, as suas intervenções são mínimas, para evitar podar a criatividade dos estudantes. As intervenções do tutor ocorrem apenas em casos extremos de distanciamento dos objetivos de aprendizagem. Em particular, na etapa final do problema podem ser necessárias intervenções mais diretas e efetivas pelo tutor para garantir que os objetivos de aprendizagem sejam atingidos.

A sessão é iniciada com a apresentação do problema, onde o tutor entrega uma cópia do texto e demais materiais de apoio do problema aos estudantes. A condução da sessão passa para o coordenador da sessão, onde a primeira ação do coordenador é realizar a leitura do texto em voz alta. Os participantes então se inscrevem junto ao coordenador da sessão para que possam expor as suas primeiras impressões sobre o problema. A sessão segue com argumentações, exposição de ideias, questionamentos e levantamento de fatos. No encerramento da sessão os participantes devem discutir metas e traçar um cronograma de trabalho para o problema. O tutor observa o desempenho dos estudantes 
na discussão e os objetivos de aprendizagem para avaliar se as metas são plausíveis e exequíveis dentro do prazo, então, caso seja necessário, são negociadas adequações nas metas e no cronograma de trabalho com os estudantes. As próximas sessões seguem o cronograma e as metas estabelecidas.

Os estudantes devem individualmente entregar uma proposta de solução para o problema, chamado de produto, até o prazo determinado no texto do problema. Alguns dos problemas foram concebidos para construção do produto em equipe, para esses problemas os estudantes se reúnem além das sessões tutorias para conceber o produto.

Além do espaço físico da sala de aula, os estudantes também são incentivados a utilizar um ambiente de aprendizagem virtual para dar continuidade à discussão do problema e como repositório para armazenar os produtos da disciplina. Está disponível no ambiente virtual um espaço para cada um dos problemas. No caso de problemas em que a construção do produto seja em equipe também é disponibilizado um espaço exclusivo para cada equipe. As opiniões dos participantes sobre suas percepções são recebidas em formulários no ambiente virtual. Os participantes são convidados a expor suas percepções sobre cada um dos problemas e sua percepção geral sobre a condução da disciplina com a utilização abordagem.

No formulário de percepções do problema os estudantes são convidados a responder 32 questões. As cinco primeiras questões são de caracterização de perfil, como idade e sexo. As próximas vinte e cinco questões são sobre as percepções dos participantes sobre a abordagem, o problema, o tutor e uma auto avaliação. Estas perguntas apresentam uma afirmação, contendo um tema ao qual se deseja avaliar a percepção, e uma escala de concordância com "discordo plenamente", "discordo" e "indiferente", considerados desfavoráveis, e "concordo" e "concordo plenamente", considerados favoráveis. As duas últimas questões são espaços abertos para que o participante inclua considerações adicionais, respectivamente, as suas percepções sobre o problema e sobre a abordagem.

Para obter as percepções gerais, os estudantes são divididos em dois grupos: no primeiro grupo são incluídos os estudantes que por algum motivo desistiram da disciplina antes da conclusão, esses são chamados de desistentes, e no segundo grupo, estudantes que concluíram a disciplina, os concluintes. Em ambos os grupos os estudantes são convidados a responder um formulário com 24 questões sobre a abordagem. Para o grupo dos desistentes se deseja obter respostas quanto aos motivos da desistência. Para o grupo dos concluintes o foco está nos benefícios identificados com a aplicação da abordagem. As cinco primeiras questões são de caracterização de perfil. As próximas dezessete apresentam uma afirmação e uma escala de concordância a exemplo do formulário sobre um problema. As últimas duas questões são espaço aberto para que os participantes descrevam suas percepções, respectivamente, sobre a abordagem e sobre a disciplina.

Para a experiência aplicada no primeiro semestre de 2016 foram propostos seis problemas $^{2}$ :

- Problema 1 - Coleção de músicas - O problema menciona um colecionador musical que deseja que novas músicas sejam criadas utilizando como base as músicas

\footnotetext{
${ }^{2}$ Agradecimentos: Gostaríamos de agradecer ao professor Martin Musicante do DIMAP da Universidade Federal do Rio Grande do Norte pelo desenvolvimento e compartilhamento dos enunciados para a confecção dos Problemas 1 e 2.
} 
de uma biblioteca fornecida. O objetivo desse problema é trabalhar os conceitos de Linguagens Formais por meio de uma equivalência dos conceitos de notas musicais com os conceitos de símbolos e cadeias.

Disponível em https://goo.gl/JgMKSp

- Problema 2 - A máquina de vender refrigerantes - O problema menciona uma empresa que deseja construir uma máquina de vender refrigerantes. O objetivo desse problema é trabalhar os conceitos de Autômatos Finitos e introduzir o conceito de não determinismo.

Disponível em https://goo.gl/zLd8ia

- Problema 3 - O controle de tráfego - O problema menciona a situação de buracos em uma estrada e solicita aos estudantes que realizem o balanceamento da proporção entre veículos leves e pesados. O objetivo desse problema é trabalhar os conceitos de Autômatos Finitos com Pilha.

Disponível em https://goo.gl/FBcT2f

- Problema 4 - O controle de tráfego - O problema é uma extensão do Problema 3. O objetivo é trabalhar os conceitos de Máquinas de Turing.

Disponível em https://goo.gl/81FHJC

- Problema 5 - Uma função bastante curiosa - O problema relata sobre a capacidade dos compiladores em identificar erros e otimizar códigos e convida os estudantes a discutirem o quanto um compilador pode ser inteligente. O objetivo é trabalhar o Problema da Parada.

Disponível em https://goo.gl/4lAspw

- Problema 6 - $\mathrm{P}$ versus NP (P = NP?) - O problema apresenta uma discussão sobre as questões de classes de problema $\mathrm{P}$ versus NP. O objetivo é trabalhar as questões de complexidade.

Disponível em https://goo.gl/X6GYkv

Na condução da disciplina, o Problema 6 foi proposto nos moldes dos anteriores, onde foi realizada uma discussão, em uma sessão tutorial única, sobre o Problema $\mathrm{P}$ versus $\mathrm{NP}$, mas não foi aplicada a abordagem PBL por completo, pois os estudantes tinham por objetivo na sessão responder uma lista de questões propostas pelo educador. Para critérios de avaliações de resultados na Seção 4 este problema não será considerado nas médias com os outros cinco problemas. O conteúdo referente a Compiladores e Gramáticas foi tratado na disciplina por meio de seminários, onde os estudantes foram separados em duplas para apresentar sobre os tópicos. Para efeitos de resultados nesse trabalho não foi realizado nenhum estudo específico com os estudantes referente aos seminários.

A avaliação de desempenho dos estudantes é progressiva. Em cada sessão o estudante é avaliado pelos critérios assiduidade e participação. São consideradas as intervenções e contribuições que o estudante traz para a sessão como participação. Esses critérios compõem, junto com a avaliação do produto, a nota do estudante para o problema. A nota final do estudante é composta pelas notas obtidas nos problemas e nas avaliações tradicionais. É interessante observar que o desempenho dos estudantes nos critérios participação e qualidade do produto se tornaram melhores à medida que eles começaram a aprender a dinâmica da abordagem. 


\section{Trabalhos correlatos}

Alguns educadores têm realizado tentativas de outras formas de abordagem, com o objetivo de transmitir de maneira mais eficaz o conhecimento e motivar os estudantes em disciplinas de Computação, e têm relatado suas experiências, assim como é realizado neste trabalho.

Para o ensino da disciplina $\mathrm{IHC}^{3}$ é apresentada uma metodologia com o objetivo de permitir aos estudantes conhecerem conceitos teóricos e técnicas sobre o desenvolvimento de interfaces a partir de uma experiência prática de construção e avaliação de projeto. Na experiência os estudantes são agrupados para construir e avaliar um projeto para a disciplina. Embora não esteja explicitado em números, está destacado que nas primeiras fases do projeto a motivação dos estudantes é baixa, mas que é crescente à medida que cresce o envolvimento com o projeto [Pelissoni and Carvalho 2003].

[Pelissoni and Carvalho 2003] mostrou um bom resultado para uma disciplina em que há um grande volume de conteúdo, um dos motivadores desse trabalho, entretanto, é necessário considerar que no caso de conceitos de Teoria da Computação o nível de abstração exigido dos estudantes é mais elevado, assim, a opção de conduzir a disciplina com apenas um projeto em todo o curso exige considerar as influências que podem ocorrer na motivação dos estudantes. Além disso, também é necessário considerar a dificuldade para que o educador construa um único projeto capaz de abordar todos os conceitos de Teoria da Computação.

Os aspectos lúdico e interativo dos jogos educacionais pode ser uma boa alternativa para auxiliar na aprendizagem e motivação dos estudantes [Silva et al. 2010]. Para o ensino de Teoria de Computação alguns trabalhos mostraram a utilização dessas ferramentas em alguma abordagem.

O trabalho [de Morais et al. 2011] tem como proposta a criação de um jogo educacional para motivação dos estudantes na aprendizagem dos conteúdos de Teoria da Computação. A ideia é um aplicativo móvel para que o estudante tenha disponibilidade de trabalhar os conceitos de forma tangencial a qualquer momento. O jogo proposto parece uma lista de exercícios e a única história descrita pode não ser suficiente para motivar o estudante.

Em [Leite et al. 2014] também é proposto um jogo educacional com um ambiente para correção automática de exercícios de Teoria da Computação. A ferramenta proposta é uma espécie de tutor virtual que foi positivamente avaliada pelos estudantes participantes, mas apresenta uma solução que contempla apenas os conteúdos de linguagens regulares e livres de contexto.

Ajudar os estudantes a compreender os conceitos de Teoria da Computação com a utilização de ferramentas também é uma abordagem utilizada. No trabalho [Vieira et al. 2003] foi criada uma ferramenta denominada de Language Emulator para que os estudantes possam manipular expressões regulares, gramáticas regulares e autômatos finitos. Um dos argumentos deste trabalho é que uma resposta mais imediata aos exercícios é um estímulo positivo para aprendizagem do estudante. Os educadores também podem utilizar a ferramenta para comparar as respostas fornecidas pelos estu-

\footnotetext{
${ }^{3}$ Interação Homem-Computador (IHC) é uma disciplina que está presente em alguns currículos de Computação com carga horária própria ou compondo a carga horária de Engenharia de Software.
} 
dantes para ter mais eficácia e agilidade nas correções, se comparado com uma correção totalmente manual. A ferramenta obteve $95 \%$ de aprovação pelos estudantes participantes do experimento. Apesar do excelente resultado descrito, apenas alguns dos conceitos de Teoria da Computação estão contemplados pela ferramenta.

O curso de Engenharia da Computação na Universidade Estadual de Feira de Santana utiliza uma abordagem com PBL desde a criação do curso em 2003. O curso possui dez módulos curriculares em que são aplicados a metodologia PBL, entre eles podem ser citados Estruturas de Dados e Engenharia de Software. Apesar de consolidado o método PBL na Universidade Estadual de Feira de Santana, não há aplicação da metodologia PBL para a disciplina de Teoria da Computação. Também é necessário considerar que a metologia PBL é aplicada institucionalmente, assim, já existe uma infraestrutura específica para realização das sessões tutoriais [dos Santos, David Moises Barreto and Burnham, Teresinha Fróes 2003].

O trabalho [Hamalainen 2004] realiza uma comparação entre aplicação de uma abordagem tradicional e uma abordagem baseada em problemas para uma disciplina de Teoria da Computação, obtendo resultados expressivos em favor da abordagem baseada em problemas, sobretudo no que diz respeito aos índices de aprovação e evasão. Entretanto, não está explícita a infraestrutura utilizada e, além disto, os autores enfatizam a necessidade de que a abordagem seja aplicada em outros contextos.

A abordagem proposta neste trabalho utilizou uma infraestrutura em sala de aula tradicional, esse é um dos seus principais diferenciais. Além disso, os artefatos produzidos neste trabalho também podem ser consolidados para que sejam utilizados em universidades como a Estadual de Feira de Santana onde já há uma infraestrutura para o PBL, mas que ainda não há módulos PBL para Teoria da Computação no curso de Engenharia de Computação.

\section{Resultados}

Todos os alunos regularmente matriculados na disciplina participaram da abordagem, mas para as pesquisas de opiniões ou interpelações sobre a abordagem foi considerado o caráter de convite. Além disso, aos estudantes não foram atribuídas quaisquer vantagens pela participação nas pesquisas.

Os participantes ${ }^{4}$ deste experimento são na maioria do sexo masculino, nove em cada dez, possuem em média 27 anos, o mais velho possui 51 anos e o mais novo possui 20 anos. A maioria dos participantes nunca desistiu da disciplina em outra oportunidade, neste número estão incluídos também aqueles que estão cursando pela primeira vez, este percentual é de $64,3 \%$. Por outro lado, falando em outras disciplinas $71,4 \%$ dos participantes já desistiram em outra oportunidade.

Um dos principais problemas enfrentados pelos educadores de disciplinas com carga teórica e matemática como Teoria da Computação é a motivação dos estudantes. Nessa experiência, na avaliação mais favorável, a percepção para $92,8 \%$ dos participantes é de que se sentiram motivados e que conseguiram atingir os objetivos de aprendizagem. $\mathrm{Na}$ avaliação menos favorável, a avaliação ficou em $71,4 \%$ na percepção de motivação

\footnotetext{
${ }^{4}$ A partir deste ponto o termo participante estará denotando apenas o estudante que respondeu a pergunta para o resultado descrito.
} 
e na percepção de atingimento dos objetivos de aprendizagem. Na média para todos os problemas, a percepção para motivação ficou em $86,2 \%$ e em $85,4 \%$ na percepção se conseguiram atingir os objetivos de aprendizagem. Os resultados para estes itens não têm objetivo de demonstrar que a abordagem possui um alto índice de aderência ou qualificar como a melhor aplicável, mas esses resultados podem ser utilizados como argumento de que a percepção dos estudantes é bem favorável. Por esse motivo essa abordagem pode ser encarada como uma alternativa para a abordagem tradicional.

A capacidade argumentativa e de sintetizar ideias é uma qualidade fundamental e esperada no estudante da área de Computação. Por esse motivo, é bastante relevante o resultado sobre a necessidade de análise e exposição clara de argumentos para elaboração de uma solução. Na avaliação dos participantes os problemas atingem integralmente o objetivo em quatro dos cinco problemas e no problema restante atinge para $85,7 \%$ dos participantes.

Trazer novos conhecimentos para os estudantes é umas das expectativas dos educadores e na percepção dos participantes este objetivo foi integralmente cumprido para três dos cinco problemas, sendo que na avaliação menos favorável o objetivo foi cumprido na percepção de $83,3 \%$ dos participantes.

Conseguir relacionar os conceitos com o mundo real é uma capacidade que permite aos estudantes propor soluções criativas e eficientes para problemas do dia a dia. $\mathrm{Na}$ percepção dos participantes os problemas que participaram se aproximam de um cenário real e atual, sendo a média favorável nessa questão de $86,1 \%$.

Uma questão bem surpreendente nesse trabalho diz respeito à percepção dos estudantes sobre o trabalho em equipe. Quando perguntados sobre a contribuição para o trabalho em equipe, o Problema 5 teve apenas $42,9 \%$ de percepções favoráveis para a questão. Os demais problemas também não foram bem avaliados na questão e a média ficou em apenas $66,5 \%$.

Na percepção dos participantes há ideias alternativas para uma mesma situação nos problemas. O Problema 5 teve a avaliação menos favorável para esse quesito, com $57,1 \%$, provavelmente porque a discussão tenha sido concentrada na contradição que o problema utiliza para atingir o objetivo de aprendizagem. Os problemas 3 e 4 atingiram integralmente percepções favoráveis dos participantes, talvez a justificativa esteja em uma discussão muito mais aberta e com diversos caminhos identificados pelos estudantes. $\mathrm{Na}$ média esse quesito teve $88,3 \%$ de percepção favorável.

No que diz respeito às referências para estudo, os participantes avaliaram que ao menos uma das referências indicadas no texto dos problemas foi utilizada, mas também destacam que foi necessário buscar outras fontes que não estavam indicadas. Em ambos os casos a média ficou em $79,7 \%$.

Sobre a utilidade dos conceitos aprendidos nos problemas para os profissionais da área de Computação, a média para a percepção favorável foi de $87,6 \%$. Entender os conceitos como úteis ajuda na motivação dos estudantes, também por esse motivo esse resultado é muito bom para avaliação do indicador de motivação.

Para que os problemas atinjam os objetivos de aprendizagem é necessário que consigam mobilizar a discussão dos estudantes durante as sessões tutoriais e instigá-los 
a continuar os estudos além das sessões. Para os participantes é quase consenso que as sessões tutoriais contribuem para o processo de resolução do problema. Na percepção dos participantes, apenas para o Problema 1 as sessões tutoriais não foram avaliadas com 100, $0 \%$ como contribuidoras para o processo de resolução do problema, obtendo $92,9 \%$. A média de $98,6 \%$, ao se considerar todos os problemas, é uma das melhores do estudo.

Outro quase consenso é no que diz respeito à percepção dos participantes se os tutores contribuem, quando necessário, para a evolução das sessões tutoriais. Os resultados são os mesmos da questão anterior.

O feedback sobre o desempenho do grupo tutorial a cada sessão também foi bem avaliado pelo participantes. É importante dar retorno aos estudantes para que eles consigam identificar o que precisam melhorar ao longo do processo e obtenham melhores resultados nos estudos. A média geral ficou em $95,2 \%$.

Em uma nota de 0 a 10, como uma avaliação geral do problema, o Problema 3 teve a melhor nota média, 7,57; a menor nota média foi 7, 00 atribuída ao Problema 5.

Entre os 25 estudantes que iniciaram a disciplina, 12 estudantes participaram de todas as atividades e 9 desses foram aprovados por conceito, enquanto 3 foram reprovados. O índice de evasão para esse semestre ficou em $52 \%$.

Algumas das percepções positivas mencionadas pelos participantes sobre a abordagem foram: "interessante", "estimula o trabalho colaborativo", "promissora", "promove soluções rápidas" e "estimula reuniões úteis".

Como oportunidade de melhoria para a aplicação da abordagem, os participantes sugeriram: (i) alternar as sessões PBL com aulas com conteúdos teóricos; (ii) calibrar a dificuldade dos problemas.

\section{Conclusão}

Os resultados dessa experiência servem como argumento de que é possível utilizar a abordagem baseada em problemas em disciplinas teóricas e obter resultados satisfatórios. A infraestrutura utilizada não é diferente do que normalmente há disponível nas instituições de ensino universitário, então, educadores que não tenham apoio institucional podem também tentar aplicar tal abordagem e obter resultados melhores que abordagem tradicional.

Os resultados dessa experiência não demonstram ganhos relevantes no que diz respeito ao índice de evasão dos estudantes, mas é necessário considerar que a abordagem foi aplicada em uma disciplina que possui um índice historicamente alto de evasão. Nessa situação, talvez fosse necessário acompanhamento ainda mais próximo desse indicador para atuar tempestivamente. Também é necessário identificar os principais motivos para que os estudantes desistam da disciplina.

A aplicação desta abordagem em outras instituições ou estudantes de perfis diferentes é necessária para avaliar os resultados em outros cenários desafiadores. A UFBA possui outros três cursos da área de Computação, além do curso de Sistema de Informação, que possuem em suas respectivas grades uma disciplina com os conceitos de Teoria da Computação, sendo assim potenciais cenários para aplicação da abordagem.

Embora os problemas tenham recebido avaliações positivas nas percepções dos 
participantes em todos os critérios avaliados, também é necessário um estudo mais detalhado incluindo a perspectiva de educadores que ainda não aplicam a abordagem proposta.

A adesão dos estudantes desistentes nas pesquisas possui grande utilidade no sentido de trazer um resultado mais completo. Desta forma, se faz necessário que os estímulos sejam suficientes para que eles respondam às pesquisas.

No que diz respeito à absorção do conhecimento pelos estudantes, também é necessário realizar um trabalho comparativo com e sem a utilização da abordagem para avaliar essa perspectiva.

Por fim, os resultados dessa experiência e o cenário desfavorável no ensino e aprendizagem tradicional de Teoria da Computação confirmam grande potencial e espaço para aplicação da abordagem PBL nesse contexto.

\section{Referências}

de Morais, D. C. S., Alencar, A. D., and de Souza, R. (2011). Jogo baseado em mlearning e aprendizado tangencial para auxílio ao ensino de teoria da computação. In Brazilian Symposium on Computers in Education (Simpósio Brasileiro de Informática na Educação-SBIE), volume 1.

Dolmans, D. H., De Grave, W., Wolfhagen, I. H., and Van Der Vleuten, C. P. (2005). Problem-based learning: Future challenges for educational practice and research. $\mathrm{Me}$ dical education, 39(7):732-741.

Hamalainen, W. (2004). Problem-based learning of theoretical computer science. In Frontiers in Education, 2004. FIE 2004. 34th Annual, volume 3, pages 20-303. IEEE.

Leite, L. S., Sibaldo, M. A. A., Carvalho, T., and Souza, R. (2014). Montanha de chomsky: jogo tutor para auxílio no ensino de teoria da computação. In XXII Workshop sobre Educação em Informática (WEI 2014), Brasília, DF.

O'Grady, M. J. (2012). Practical problem-based learning in computing education. ACM Transactions on Computing Education (TOCE), 12(3):10.

Pelissoni, C. G. and Carvalho, J. d. (2003). Uma proposta de metodologia para o ensino da disciplina interação humano-computador em cursos de computação e informática. In XI Workshop sobre Educação em Informática (WEI 2003), Campinas, SP, volume 11.

dos Santos, David Moises Barreto and Burnham, Teresinha Fróes (2003). O pensamento de Paulo Freire e PBL: primeiras aproximações e afastamentos. In XI Workshop sobre Educação em Informática (WEI 2003), Campinas, SP.

Silva, R. C., Binsfeld, R. L., Carelli, I. M., and Watanabe, R. (2010). Automata defense 2.0: reedição de um jogo educacional para apoio em linguagens formais e autômatos. In Brazilian Symposium on Computers in Education (Simpósio Brasileiro de Informática na Educação-SBIE), volume 1.

Vieira, L. F. M., Vieira, M. A. M., and Vieira, N. J. (2003). Language emulator, uma ferramenta de auxílio no ensino de teoria da computação. In XIII Workshop sobre Educação em Computação-XXV Congresso da Sociedade Brasileira de Computação.

Wood, D. F. (2003). Problem based learning. BMJ: British Medical Journal, 326(7384):328. 